\title{
ErgoMonitor: Ambiente de Monitoramento da Usabilidade de Web Sites Analisando Arquivos de Log
}

\author{
André Luís Schwerz', Marcelo Morandini', Walter de Abreu Cybis ${ }^{2}$ \\ 1Departamento de Informática - Universidade Estadual de Maringá (UEM) \\ Av. Colombo, 5790 - Bloco 19 - Centro - CEP 87020-900 - Maringá - PR - Brasil. \\ ${ }^{2}$ Departamento de Informática e Estatística - Universidade Federal de Santa Catarina \\ Caixa Postal 476 - Campus Universitário - Trindade - Florianópolis - SC - Brasil. \\ \{als, morandin\}@din.uem.br; walter.cybis@polymtl.ca
}

\begin{abstract}
This paper presents the definition of the ErgoMonitor and its implementation initial view, an web sites usability automatized evaluation environment that implements a task oriented log files analysis approach. This environment collects data that are stored at the server log files and calculate usability taxes and metrics. The results produced by the ErgoMonitor are not influentiated by subjectivities or observations problems. On the other hand, these results are dependant on users explorative behaviours that can be only visiting the web site. The ErgoMonitor is indicated for continuos monitoring studies, where usability taxes and metrics for same tasks can be compared with theirs historical averages, to identify normality deviances.
\end{abstract}

Resumo. Este artigo apresenta a definição e uma visão inicial da implementação do ErgoMonitor, um ambiente de avaliação de usabilidade em web sites baseado na análise de tarefas nos arquivos de log. Este ambiente coleta dados que estão armazenados nos arquivos de log do servidor e calcula métricas e taxas de usabilidade. Os resultados produzidos pelo ErgoMonitor não são influenciados por efeitos subjetivos. Porém, estes resultados são dependentes dos comportamentos de exploração dos usuários que visitam o web site. $O$ ErgoMonitor é indicado em monitoramentos contínuos, onde metas e taxas de usabilidade para uma mesma tarefa podem ser comparadas através de médias históricas, identificando desvios de normalidade.

\section{Introdução}

A usabilidade, conforme a Norma ISO/IEC 9241, pode ser definida como: efetividade, eficiência e satisfação com que cada usuário específico alcança suas metas definidas em ambientes particulares. Efetividade significa a precisão e perfeição com que usuários podem alcançar metas em ambientes particulares. Eficiência significa os recursos gastos em relação à precisão e perfeição de metas alcançadas e a satisfação significa o conforto e aceitabilidade do sistema de trabalho para seus usuários e outras pessoas afetadas por seus usos [ISO/DIS 9241, 1997].

Ainda, esta Norma apresenta medidas de usabilidade que devem ser baseadas em dados que reflitam o contexto do usuário interagindo com um determinado produto, no caso web sites, em seu ambiente específico de trabalho. Assim é possível coletar dados através de meios objetivos fornecendo indicações de eficácia e eficiência e por meios 


\section{Simpósio Brasileiro de Qualidade de Software}

subjetivos gerando medidas que estão ligadas diretamente com a satisfação do usuário. Assim, para avaliar a usabilidade de um sistema interativo algumas particularidades devem ser analisadas [LabIUtil, 2003]:

- validar a eficácia da interação humano-computador, face a efetiva realização das tarefas por parte dos usuários;

- verificar a eficiência desta interação, face os recursos empregados (tempo, quantidade de incidentes, passos desnecessários, busca de ajuda, etc.); e

- obter indícios da satisfação ou insatisfação (efeito subjetivo) que ela possa trazer ao usuário. Estes objetivos devem ser pensados em relação aos diferentes contextos de operação previstos para o sistema.

Desta maneira pode-se elucidar os objetivos de uma avaliação de usabilidade [LabIUtil, 2003]:

- constatar, observar e registrar, problemas efetivos de usabilidade durante a interação;

- calcular métricas objetivas para eficácia, eficiência e produtividade do usuário na interação com o sistema;

- diagnosticar as características do projeto que provavelmente atrapalhem a interação por estarem em desconformidade com padrões implícitos e explícitos de usabilidade;

- prever dificuldades de aprendizado na operação do sistema;

- prever os tempos de execução de tarefas informatizadas;

- conhecer a opinião do usuário em relação ao sistema; e

- sugerir as ações de re-projeto mais evidentes face os problemas de interação efetivos ou diagnosticados.

Em confronto com a necessidade de resolver problemas de usabilidade em interfaces web, além de procurar conhecer seus usuários, sugere-se a adoção de uma metodologia que prevê o monitoramento da interação dos usuários que interagem com a interface como forma de descobrir informações sobre a usabilidade. Esta metodologia prevê, portanto, um modelo evolucionário do usuário com base em atributos que podem ser coletados durante a interação.

Algumas técnicas de avaliação de usabilidade em web sites [Bastien e Scapin 1998], [The Bobby Tool 2004], [Brajnik 2000], [WebTrends 2005], [W3C 2003], [Cybis et. alli. 2002], [Scapin et. alli. 2001] abordam aspectos qualitativos da interface gerando resultados com problemas de subjetividade. Este artigo apresenta uma avaliação de modo objetiva que visa quantificar a eficiência, a eficácia e a satisfação do usuário quando interagir em um web site para realizar tarefas pré-definidas e isto é verificado através dos registros nos arquivos de log do servidor. Nós desejamos demonstrar que é possível através da análise de arquivos de log apoiar os estudos sobre usabilidade. Usando a mineração dos dados dos arquivos de $\log$, é possível obter rapidamente as taxas e as métricas que quantificam a usabilidade de determinadas tarefas, produzindo avaliações confiáveis, objetivas e de baixo custo. 


\section{Simpósio Brasileiro de Qualidade de Software}

Algumas ferramentas de análise de log estudadas, e usadas como base para este trabalho, apresentam características que podem ser divididas em [Brajnik, 2000]:

- Técnico (o endereço de onde os acessos vieram, urls e documentos acessados, frequiência e duração do acesso a diferentes páginas);

- Perspectiva de interação (requisições de páginas e documentos, data e hora dos acessos, navegador e sistema operacional usado, entre outros); e

- Perspectiva de uso (tipo e número de erros, solicitações de ajuda, componentes com erros, etc).

Esta abordagem é especialmente útil para B2B (Business to Business), ERP (Electronic Resource Planning) ou alguns web sites específicos para aplicações intranet, onde a incidência transações de tarefas inferidas é grande e o acesso do usuário é controlado. Em particular, espera-se que os resultados obtidos nestes casos sejam muito precisos. Isso não acontece em casos de web sites de comercio eletrônico B2C (Business to Consumers), onde os resultados são influenciados pela existência de agentes de software e de usuários, que visitam o web site com outros objetivos, com o intuído de somente conhecer seu conteúdo. Além disso, as páginas podem ser armazenadas na memória cache da máquina do cliente, de maneira que não haverá registro no arquivo de log do servidor referente a um novo acesso. Mesmo assim, a análise de dados de tarefas orientadas pode ser útil, se seu resultado for elaborado de maneira seletiva, isto é, comparado com os valores do histórico obtido no passado para as mesmas condições de contexto. Neste momento, o foco deve estar voltado preferivelmente para um distúrbio do nível de usabilidade. Assim, um projetista web pode, rapidamente, identificar uma curva de distúrbio nesse nível de usabilidade causado, possivelmente, pelo acesso com problemas usabilidade em alguma interface que tenha sido acessada algum tempo atrás.

\section{O ErgoMonitor}

Este ambiente, que realiza a análise de tarefas orientadas pelo arquivo de $\log$ do servidor, foi denominado ErgoMonitor [Morandini e Cybis 2003] e é capaz de apresentar taxas e métricas que quantificam a usabilidade de tarefas de um web site. A usabilidade é verificada pelo monitoramento, quando interações reais forem realizadas por usuários reais acessando um web site em um ambiente real, com suas condições especiais e suas limitações. Isso define o foco de atenção das avaliações de usabilidade: problemas de usabilidade que ocorrem efetivamente, sem que se tenha o conhecimento pleno das condições de contexto de uso envolvidas com tais problemas. As taxas e métricas calculadas baseadas nas informações coletadas de acessos (reais) de um web site são automatizadas e invisíveis ao usuário. Após o cálculo, as taxas e métricas de usabilidade verificadas podem ser comparadas com valores históricos da mesma tarefa ou de outra em um web site similar, e os resultados obtidos não sofrem influências externas, tais como, ruídos durante a interação ou interferências do observador. $\mathrm{O}$ ErgoMonitor pode somente ser usado em avaliações de usabilidade em web sites, permitindo a definição de métricas de usabilidade produzidas por taxas, tais como: solicitação de ajuda; apresentação de mensagem de erro; repetição de tarefas (isto é, uma taxa que represente quantas vezes uma tarefa teve que ser repetida); taxa de desistência e cancelamento. 


\section{Simpósio Brasileiro de Qualidade de Software}

A arquitetura conceitual do ErgoMonitor pode ser apresentada como:

- Pré-Diagnósticos (opcional) - obtido como resultado de técnicas diagnósticas capazes de identificar possíveis problemas em uma interface que apóiam determinadas tarefas. Por exemplo: em consequiência da falta de condução em uma interface, o usuário pode ser levado a hesitações, a busca de ajuda, a ficar inativo ou a cometer erros;

- Modelo de Tarefas e Comportamento a ser Monitorado - baseado em um diagnóstico quantitativo e qualitativo e nas características do web site, o avaliador deve elaborar um modelo de tarefas nas quais os comportamentos de sucesso, desistência, cancelamento, abandono, busca de ajuda e ao enfrentamento de erros sejam identificados de forma clara e objetiva através da análise de arquivos de log;

- Taxas de Usabilidade Verificadas - calculadas através de ferramentas de filtragem e mineração de dados do arquivo de $\log$ do servidor. Estas taxas quantificam as características da interação. Exemplos são: taxa de sucesso no preenchimento de um formulário, taxa de desistência em uma pesquisa, etc;

- Parâmetros de Usabilidade - a eficiência, desistência, solicitação de ajuda, mensagens de erros, repetição de tarefas, entre outras, são comparadas com valores históricos de taxas e métricas de usabilidade relacionados à mesma tarefa. As diferenças significativas entre esses valores podem facilmente ser identificadas e algumas ações emergenciais ou uma manutenção pode ser executada rapidamente. Um exemplo dessas ações seria recuperar a configuração da versão anterior do web site.

A proposta de avaliação relacionada ao ErgoMonitor é específica para análises de web sites por duas razões: a estratégia de análise de arquivos de log do servidor é de natureza particular desse tipo de ambiente. De fato, um web site é distinto por sua manutenção dinâmica. A maioria dos web sites geralmente possuem determinadas ações de manutenção e não é comum encontrar equipes responsáveis por essas ações [Nielsen 2000].

No contexto do ErgoMonitor, as tarefas que podem ser executadas pelos usuários na interação com o web site devem ser consideradas "objetivas" ou "fechadas". Estas tarefas especiais possuem uma característica determinante: apresentam pontos (ou seja, urls) de início e fim bem definidos. Assim a url final que pode ser acessada pelo usuário ao executar uma tarefa, e ao conseguir o sucesso deve ser especificada como url de sucesso. Por essa mesma razão, a url inicial é provavelmente a homepage para a maioria das tarefas suportadas pelo web site. Neste contexto, se os usuários, que somente desejam visitar o web site e não concluem uma tarefa, não são considerados, e as métricas de eficiência e eficácia podem ser calculadas de modo objetivo e sem ambigüidade devido ao uso e mineração dos dados do arquivo de $\log$ do servidor.

O foco inicial do ErgoMonitor são os web sites de comércio eletrônico que caracterizam-se pelo uso dos recursos da internet para agilizar transações comerciais e aumentar a eficiência dos processos nas organizações. Desta maneira, as principais tarefas apoiadas por este domínio de aplicação constituem-se de tarefas "objetivas" ou "fechadas". Assim, podemos identificar no escopo dos web sites de comércio eletrônico tarefas como: localização de produtos, elaboração de pedidos, efetivação dos pagamentos, vendas em geral, etc.[Meira Jr. et alli, 2002]. 
O avaliador-operador do ErgoMonitor deve ter uma participação ativa na configuração do ambiente, uma vez que ele deve definir um parâmetro de "serviço" como o endereço do arquivo de log do servidor. Também, o avaliador-operador deve modelar as tarefas e os comportamentos. Esta modelagem é, basicamente, apresentar um conjunto de urls que serão acessados quando uma tarefa for executada. Assim, nos modelos é necessário incluir urls de sucesso, desistência, solicitação de ajuda, mensagens de erro, etc.

A Figura 1 ilustra uma visão geral das atividades do ErgoMonitor: coleta de dados do arquivo de $\log$ do servidor, geração de taxas e métricas e apresentação de laudos do monitoramento. A Figura 2 ilustra o funcionamento do Log Control que é constituído por um conjunto de programas responsáveis de filtrar os arquivos de log e remover informações que não são significantes para o monitoramento da usabilidade (tal como arquivos .gif). Também, uma outra entrada do Log Control é o Modelo de Tarefas e Comportamentos a Monitorar. O avaliador-operador é responsável de gerar este modelo, usando como base urls do web site e um eventual diagnóstico precedente dos problemas de usabilidade (que pode ser o resultado de uma tradicional técnica avaliação de usabilidade). Obviamente, o Modelo de Tarefas e Comportamentos a Monitorar deve ser composto por tarefas "objetivas" ou "fechadas", que possuem um começo e fim claramente bem definidos, e, a cada uma delas, estarão associados comportamentos indicativos de eficiência e de eficácia.

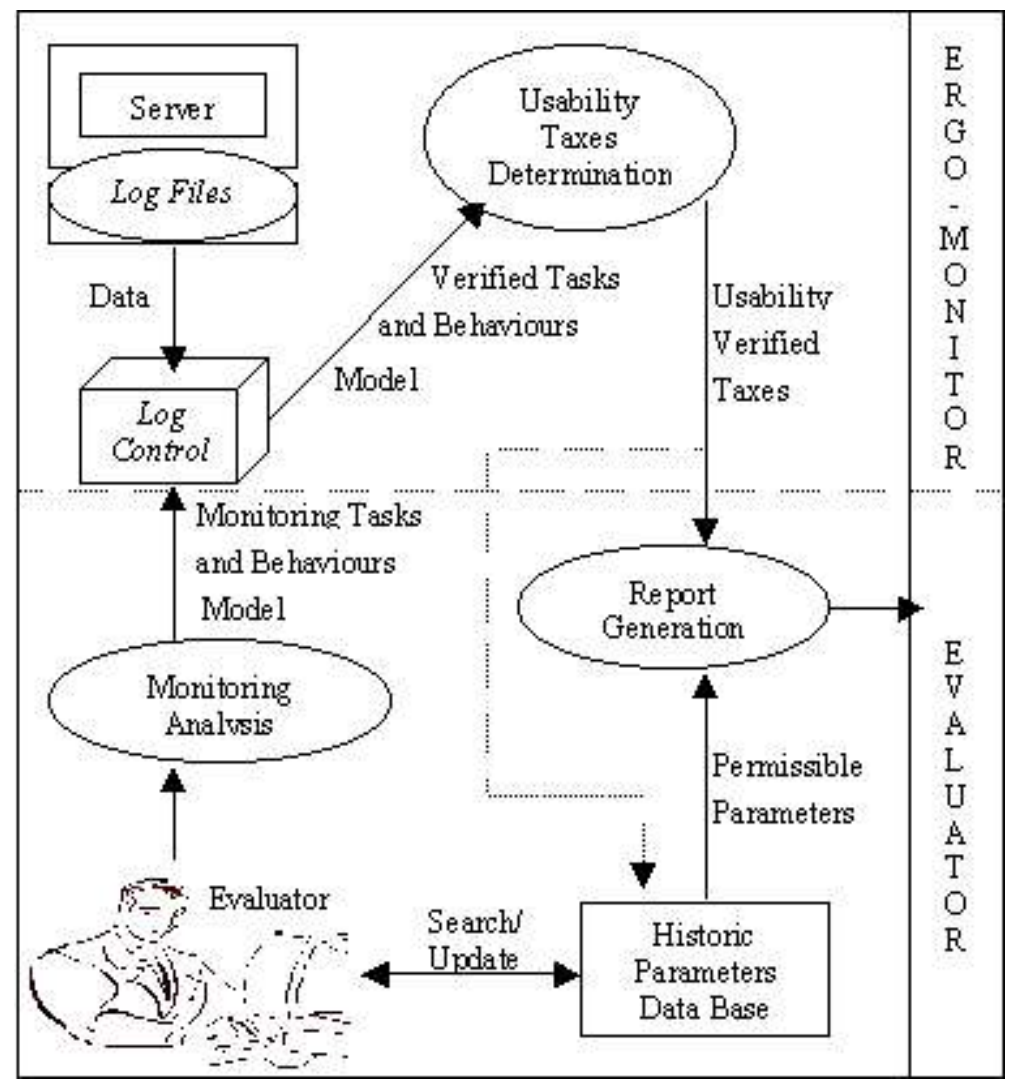

Figura 1 - Visão Geral do ErgoMonitor 


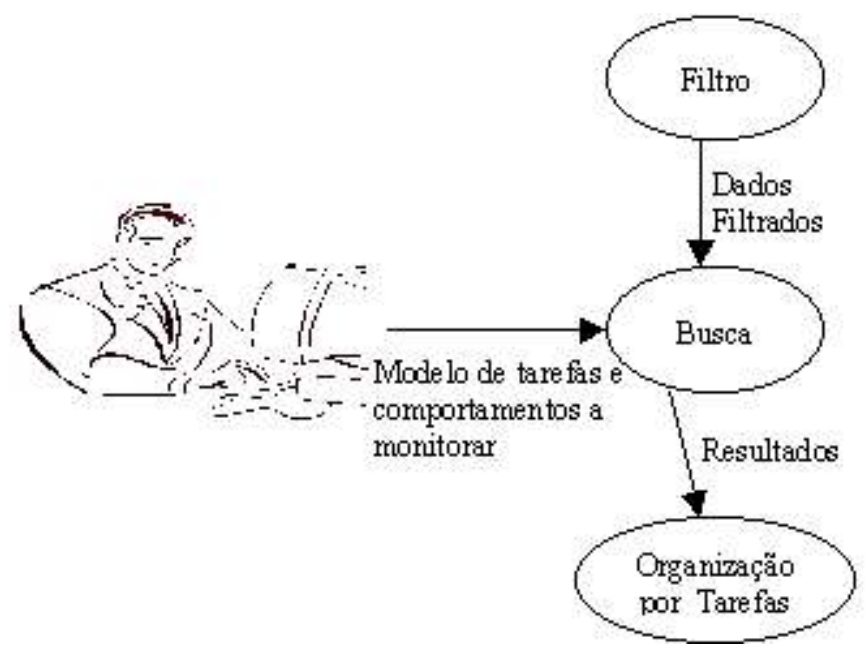

Figura 2 - Descrição do Log Control

O resultado previsto do Log Control é uma contabilidade da ocorrência de cada comportamento das tarefas envolvidas e produzir um Modelo de Tarefas $e$ Comportamentos Verificados. Este modelo é a base para determinação de taxas e métricas. Conseqüentemente, neste contexto, uma taxa é uma medida intermediária da qualidade de interação (sucesso, desistência, cancelamento, etc) e uma métrica representa uma medida de usabilidade como foi estabelecido pela ISO/IEC 9241 [ISO/DIS 9241 1997] e, no contexto do ErgoMonitor, é calculada a partir da produção de taxas. O ambiente ErgoMonitor é baseado na divisão do trabalho: a "máquina" faz a coleta e análise dos dados do arquivo de $\log$ e o avaliador-operador realiza a modelagem de tarefas e comportamentos para ser monitorado e é também responsável pela geração de relatórios.

Assim, o ErgoMonitor é definido como uma ferramenta avançada de análise de log que especializa serviços que possam caracterizar a "análise de tarefas inferíveis" em vez de apresentar uma análise de rastros dos clicks ("clicksstreams analysis")[Keynote 2004]. Espera-se a produção de "taxas e métricas de usabilidade" no lugar de informações sobre o "retorno de investimento" relacionado a possíveis manutenções no web site.

Nos próximos parágrafos nós apresentamos algumas características da visão inicial da implementação do ErgoMonitor:

Formulários: Eles referem-se à necessidade de apresentar importantes parâmetros relacionados ao monitoramento atual, tais como: endereço do arquivo de $\log$, web site, dados de acesso do arquivo de $\log$, lista de tarefas inferidas; identificação das tarefas; páginas que compõem as tarefas (incluindo a página inicial - url, páginas intermediárias - sequiência de urls, página final - url, páginas de ajuda - conjunto de urls e páginas de erros - conjunto de urls); listas de interfaces (incluindo a identificação da versão e data que estavam disponíveis aos usuários). Com esta estrutura, um web site é visto como uma coleção de tarefas, e cada uma delas estão baseadas em uma coleção de urls. Cada web site pode possuir uma lista de arquivos de log, previamente filtrados pelo avaliador-operador, onde as tarefas poderão ser monitoradas. Além disso, um avaliador-operador pode manipular diversos web sites, como é apresentado na Figura 3. O ErgoMonitor estará monitorando a usabilidade em uma estrutura de tarefas pouco 
variáveis, que definem interfaces muito variáveis. Idealmente, as descrições das tarefas são informadas somente a cada vez que o web site é revisado.

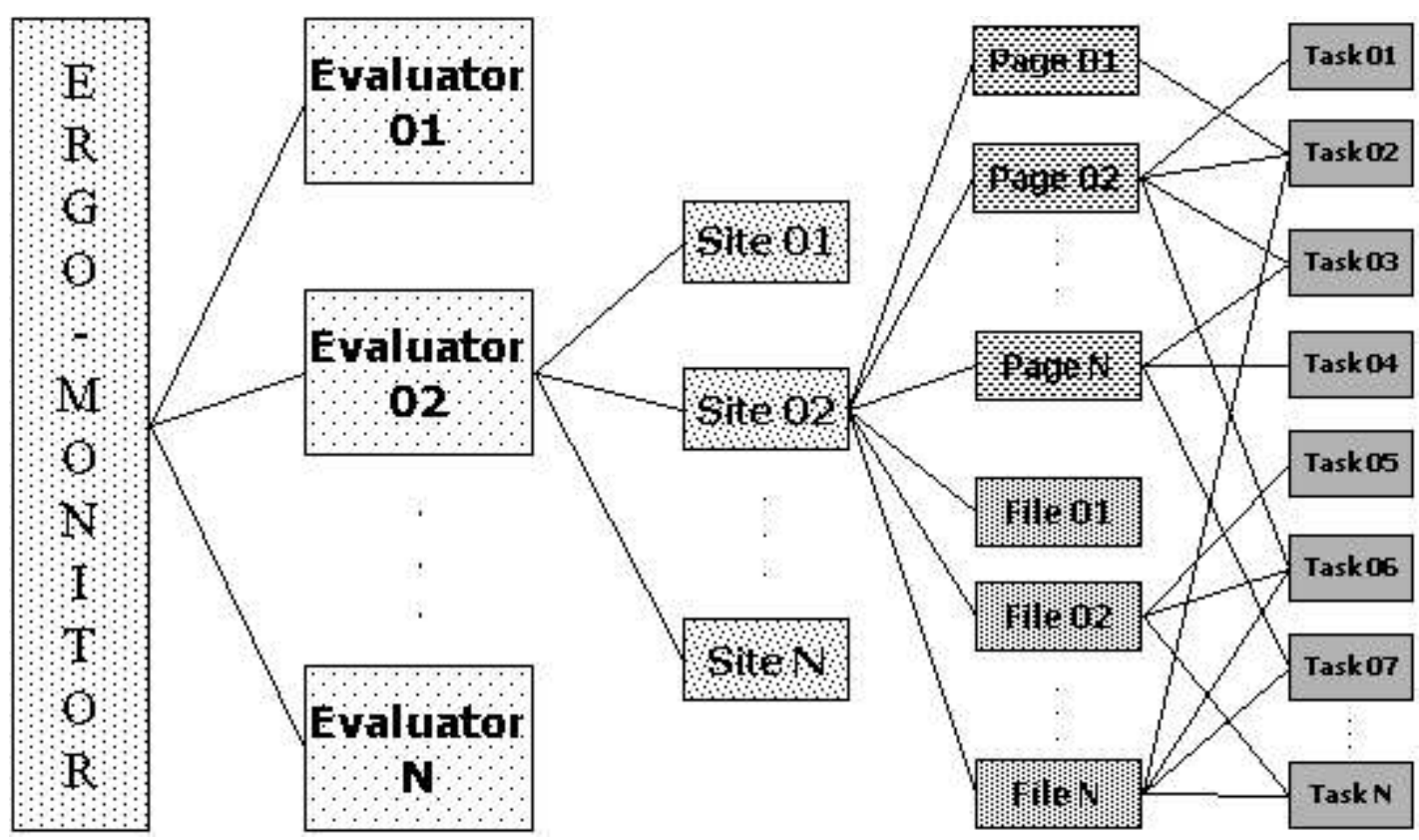

Figura 3 - Organograma do ErgoMonitor

Núcleo Funcional: monta o conjunto de comportamentos associados à realização de cada tarefa inferida declarada nos formulários de monitoramento. Mesmo sendo fácil a apresentação de vários outros comportamentos, o ErgoMonitor monitorará especificamente:

- Comportamento Bem Sucedido = url inicial + url intermediária + url final;

- Sucesso com Erro = url inicial + url intermediária + todas $u r l s$ de erro + url final;

- Sucesso com Ajuda = url inicial + + url intermediária + url de ajuda;

- Abandono = url inicial $+u r l$ intermediária (opcional) + exceto $u r l$ final; e

- Sucesso com Repetições = n Abandonos + Comportamento bem sucedido.

Após esse passo, este módulo filtrará o comportamento nos arquivos de log do servidor e computará taxas e métricas de usabilidade listados nas seções seguintes.

Relatórios: para produzir os resultados esperados (isto é, relatórios) o ErgoMonitor requer métricas de usabilidade armazenadas de acordo com os parâmetros selecionados pelo avaliador-operador. Por padrão, os relatórios apresentarão diversas métricas de usabilidade correspondente a uma tarefa e possibilitará diferentes versões de interfaces associadas. O ErgoMonitor fornecerá aos desenvolvedores do web site:

\section{Taxas de Usabilidade}

- Quantidade de Comportamentos Bem Sucedidos (BS)

- Quantidade de Tarefas Verificadas (TV)

- Quantidade de BS com Ajuda (SA)

- Quantidade de BS com Erro (SE)

- Quantidade de BS com Repetição (SR) 


\section{Métricas de Usabilidade}

- Medida de Eficiência $=\frac{B S}{T V}$

- Tempo Médio da Tarefa $=\frac{\sum \text { tempoBS }}{B S}$

- Incidentes na Taxa de Sucesso $=\frac{S A+S E+S R}{B S}$

Nós desejamos mostrar que é possível através da análise dos arquivos de log apresentar estudos de usabilidade: usando a mineração de dados do arquivo de $\log$ é possível obter taxas e métricas que quantificam níveis de usabilidade de determinadas tarefas, produzindo avaliações confiáveis, objetivas, rápidas e de baixo custo.

\section{1 - Uma visão inicial da implementação do ErgoMonitor}

Um protótipo do Ergo-Monitor foi desenvolvido utilizando a ferramenta RAD (Rapid Application Development) Borland Delphi 6.0 [William 2004] beneficiando-se de vantagens para produzir, rapidamente, sistemas interativos. Nós também utilizamos o Paradox [World Class Software Solutions 2004] para o armazenamento dos dados, por se tratar de um sistema de banco de dados relacional baseados em tabelas fornecendo tecnologia suficiente para o desenvolvimento do protótipo.

O desenvolvimento do protótipo do Ergo-Monitor foi dividido em cadastros de web site, páginas, tarefas e usuários e identificação dos arquivos de log. Estas etapas serão detalhadas abaixo.

\subsection{1 - Cadastros de Web site, Páginas, Tarefas e Usuários}

Com a finalidade de coletar dados importantes para a avaliação o ErgoMonitor fornece ao avaliador-operador diálogos para cadastro de web sites, páginas, tarefas e usuários. A respeito do cadastro de web site, o avaliador-operador deve informar o web site que será avaliado e os dados relevantes no momento são: título do web site e descrições breves dos objetivos. No cadastro de páginas que compõe o web site, o avaliador-operador deve fornecer os seguintes dados: a url da página; um rótulo que servirá como um título da página em operações posteriores; uma descrição sobre as suas funcionalidades e particularidades.

Um conjunto de páginas compõe um registro de páginas e as tarefas que o web site suporta compõem um registro de tarefa. Como o ErgoMonitor basicamente suporta tarefas "fechadas" ou "objetivas", cada uma delas deve ter uma página (url) de início e de fim. O relacionamento entre essas e outras páginas de uma tarefa também devem ser informadas, produzindo um registro de transação. $\mathrm{O}$ cadastro de usuários é meramente uma funcionalidade administrativa do sistema, o qual visa controlar os usuários (avaliador-operador) para que informações, a respeito dos web sites de um determinado usuário, não sejam manipuladas por outro. 


\subsection{2 - Identificação do Arquivo de $\log$ do Servidor}

O avaliador-operador deve informar o(s) arquivo(s) de $\log$ do servidor. O sistema produzirá uma cópia deste(s) arquivo(s), gerando um arquivo temporário que será utilizado na avaliação. O ErgoMonitor processará o arquivo temporário identificando e comparando as urls do arquivo com as informadas pelo avaliador-operador no cadastro de páginas. A confirmação dessa comparação irá gerar um registro no banco de dados. Caso uma url que não pertença ao registro de páginas, esta será eliminada, uma vez que não pertence ao contexto da avaliação.

\subsection{3 - O Banco de Dados do ErgoMonitor}

Para implementar o ambiente do ErgoMonitor, tornou-se necessário ao projeto o uso de uma base de dados. Assim, a Figura 4 apresenta esta descrição da base de dados e as tabelas presentes nesta figura são:

- User - Armazena os diferentes usuários (avaliador-operador);

- Site - Armazena os dados do web site de um determinado usuário;

- Page - Armazena as páginas de um determinado web site;

- Task - Armazena as tarefas de um determinado web site;

- Page_Task - Armazena as páginas de uma determinada tarefa;

- Transaction - Armazena as transações entre as páginas de uma determinada tarefa;

- File - Armazena os dados do arquivo de log de um determinado web site; e

- File_Page - Armazena a seqüência de acessos de páginas de um determinado arquivo. 


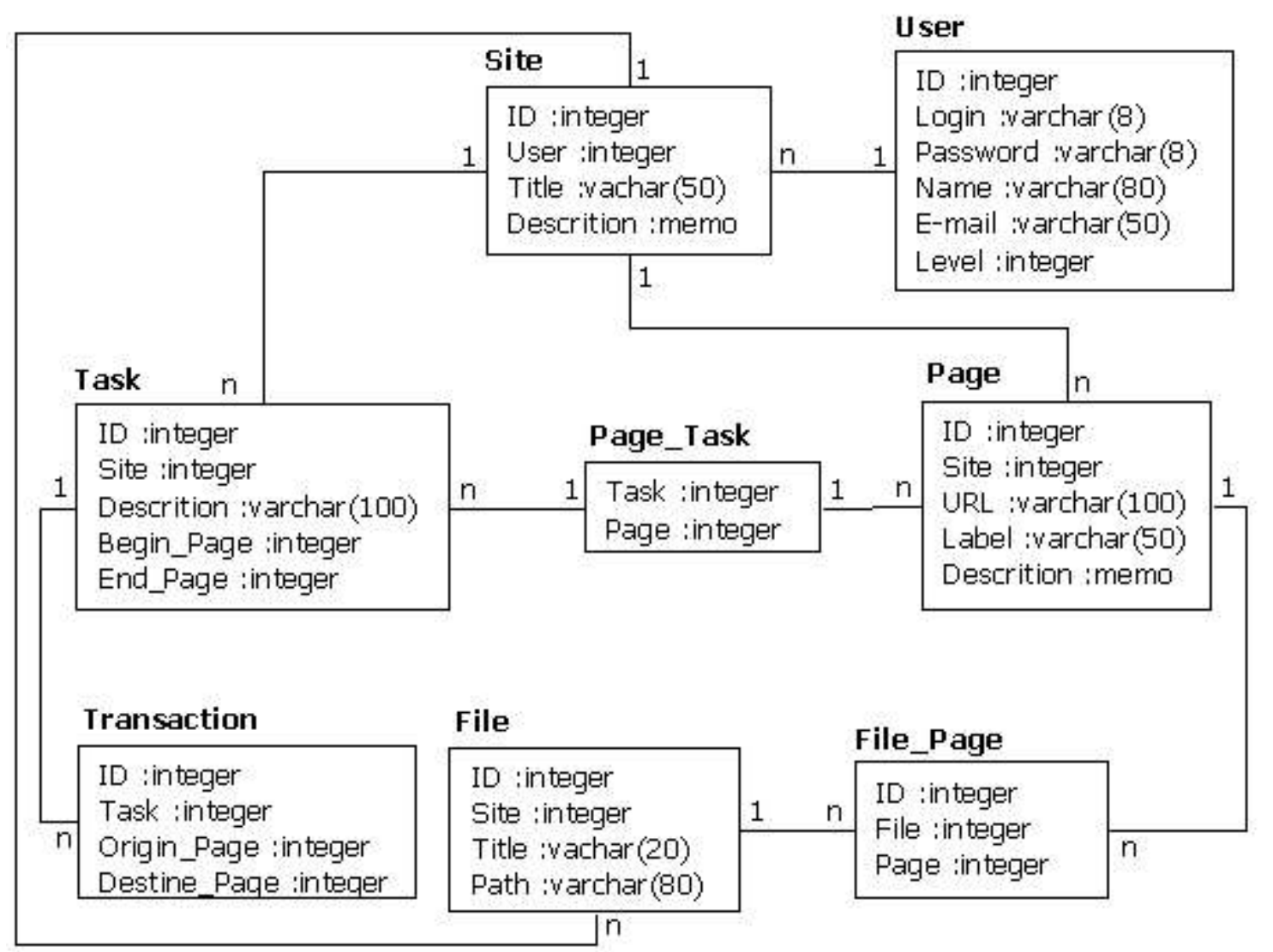

Figura 4 - Descrição do Banco de Dados do ErgoMonitor

\section{Conclusão}

Este artigo apresenta, de maneira geral, a proposta sobre o monitoramento automático de usabilidade em web sites. O ErgoMonitor é o ambiente que dá apoio a esse monitoramento, baseado nas interações armazenadas em registros nos arquivos de log do servidor. Este ambiente tem uma importante característica: apresenta resultados que não são influenciados por interferências e ruídos usuais, os quais são comuns em técnicas de avaliação baseadas nas observações com os usuários.

Assim, esta proposta, e o ambiente que apóia isto, pode somente ser aplicado para a análise de tarefas fechadas e objetivas. Estas tarefas são especificadas por urls de início e fim, e o acesso a uma url de início indica que o usuário deseja atingir um objetivo. Devido ao fato de um web site ter uma grande quantidade de usuários que desejam somente o visitar e não desejam atingir nenhum objetivo especifico, como a compra de algo ou adquirir uma informação particular, os resultados obtidos pelo ErgoMonitor devem ser analisados com dados de medidas históricas previamente armazenados. Estes dados podem ser obtidos com avaliações de usabilidade de web sites similares que implementam o mesmo objetivo sob a avaliação. Conseqüentemente, a usabilidade de um web site pode ser identificada pelos desenvolvedores, de uma maneira fácil, em situações onde as soluções de interfaces não são boas ou os usuários não são capazes de interagirem corretamente. Os resultados são quantitativos, de baixo custo e obtidos de maneira rápida e sistemática. Eles referem-se a usuários e interações, mas também, podem ser vistos como um resultado limitado quando comparado com propostas de avaliação de usabilidade que empregam técnicas e abordagens consideradas "tradicionais". De fato, uma perspectiva de interação é tão nula para os 
objetivos de análise de usabilidade quando nós não conhecemos os objetivos dos usuários ao interagem com um web site.

Finalmente, é importante informar, que o ErgoMonitor pode ser facilmente adaptado para apoiar uma ferramenta de testes para a avaliação de usabilidade sob observação de usuários. Sob o controle dessas situações, os avaliadores podem conhecer seus usuários (ou seja, IPs), quando estes interagem a fim de alcançar seus objetivos. Assim, o modelo de comportamento previsto enquanto as interações com o web site são realizadas permitem definições automáticas de taxas e métricas de usabilidade. Também, este ambiente pode evitar (ou, ao menos, minimizar) o uso de todos os equipamentos necessários para uma observação, tal como câmeras de vídeo, monitores, gravadores, etc. Também, o ErgoMonitor pode ser usado como uma ferramenta que apresenta informações adicionais sobre a usabilidade do web site e, em situações onde o avaliador considerar importante para conduzir as avaliações utilizar técnicas e métodos tradicionais.

\section{Referências}

Bastien, J. M. C., Scapin, D. L. (1998) "A Validation of Ergonomic Criteria for The Evaluation of Human-Computer Interfaces"; International Journal of HumanComputer Interaction, 4, 183-196.

Brajnik, G. (2000) “Automatic Web Usability Evaluation: What Needs To Be Done”, In Proc. of 6th Human Factors and the Web Conference, Austin, Texas - EUA, Junho, 2000.

Cybis, W.A., Scapin, D. L. ; Andres, D.P., Morandini, M. (2002). "ErgoCoIn : An usability Inspection Tool for Web Sites Evaluation", INRIA repports.

ISO/DIS 9241 (1997); "Dialogue Principles in Guiding the Evaluation of User Interfaces", Guidance on Usability.

Keynote (2004). "Research Manager Clickstream", disponível em http://www.keynote.com/solutions/solutions cem research mgr clickstream.html acessado em setembro de 2004

LabIUtil (2003) "Laboratório de Utilizabilidade", Departamento de Informática, Universidade Federal de Santa Catarina (UFSC), disponível em http://www.labiutil.inf.ufsc.br; acessado em abril de 2003.

Meira Jr., W.; Murta, C.D., Campos S.V.A.; Guedes Neto, D. O. (2002); Sistemas de Comércio Eletrônico - Projeto e Desenvolvido; Editora Campus.

Morandini, M., Cybis, W.A. (2003) "Definition and Prototyping of ErgoMonitor". In: Proccedings of International Conference on Human-Computer Interaction, Creta. v.II, p. 1396-1399.

Nielsen, J.(2000); “Designing Web Sites - Designing Web Usability”, Editora Campus.

Scapin, D., Leulier, C., Vanderbonckt, J., Mariage, C., Bastien, C, Palanque, P., Farenc, C., Bastilde, R.(2001) "Towards Automated Testing of Web Usability Guidelines", In. Tools for Working with Guidelines London Springer, pp. 293-304.

The Bobby Tool (2004), disponível em www.cast.org, acessado em maio de 2004. 
WebTrends (2005)."WebTrends7, (n.d.)", disponível em http://www.webtrends.com, acessado em fevereiro de 2005

William, B. (2004) "Borland Delphi 6 Inprise" - Serie IT Educational; Alta Books

World Class Software Solutions (2004) "Paradox Products and Services"; disponível em http://www.fmsinc.com/paradox/index.html, acessado em janeiro de 2004.

World Wide Web Consortium's (W3C) (2002) "Web Accessibility Initiative", disponível em http://www.w3c.com, acessado em maio de 2002. 